an NGS panel, identifying challenges in case classification and possible solutions.

Methodology We performed the FoundationOne CDx NGS panel on $60 \mathrm{EC}$ and assigned molecular subtype: POLE mutated (POLEmut), mismatch repair deficient (MMRd), p53 abnormal (p53abn) or no specific molecular subtype (NSMP). Result(s)* In 55 patients the molecular classification was successful. A pathogenic POLE mutation was detected in 9 cases (POLEmut). 20 were MMRd (12 MSI-high, 8 MSI-indeterminate based on the NGS panel MSI classifier) and a known or likely MMR gene mutation was found in 7 of these. Of the remaining 26 cases, 17 carried a TP53 mutation (p53abn) and the remaining 9 were considered to be NSMP. The tumor mutation burden $(\mathrm{TMB})$ was significantly different $(\mathrm{p}<0.001)$ in the molecular subtypes (A) and high TMB ( $>55$ mut/MB) was $100 \%$ specific for POLEmut EC $(\mathrm{p}<0.001)$. High TMB was specific for known pathogenic POLE mutations and was not elevated in cases with solely non-pathogenic POLE mutations (B).

In non-POLEmut cases, TMB was higher in MSI-high and MSI-indeterminate than microsatellite stable (MSS) cases (C), and a TMB of $>7$ mut/MB was 100\% specific for MMRd EC. There was one MSS EC with a TMB of $18 \mathrm{mut} / \mathrm{MB}$ but it showed loss of MLH1 and PMS2 proteins on immunostaining and was classified as MMRd.

Conclusion* An NGS panel can be used in the molecular classification of EC when there is sufficient tumor cellularity. TMB can be used as an adjunct in molecular subtype diagnosis for tumors that are difficult to classify. Additionally, TMB can potentially serve as a diagnostic adjunct in cases with POLE mutation of unknown significance.

\section{THE ROLE OF GENITAL TRACT MICROBIOTA CONTINUUM IN ENDOMETRIAL MALIGNANCY}

1;2A Semertzidou*, 'D Macintyre, '1 Marchesi, 1;2p Bennett, 1;2;3M Kyrgiou. 'Imperial College London, Metabolism, Digestion and Reproduction, London, UK; ${ }^{2}$ Queen Charlotte's and Chelsea- Hammersmith Hospital, Imperial Healthcare NHS Trust, London, UK; ${ }^{3}$ Imperial college London, Metabolism, Digestion and Reproduction, London, UK

\subsection{6/ijgc-2021-ESGO.223}

Introduction/Background* Endometrial cancer has a dominant place among gynaecological cancers and is the fourth most common malignancy in women. Accumulating reports have associated gynaecological precancer and cancer with dysbiotic microenvironments. Our aim was to identify a microbial signature in endometrial cancer and explore its role in disease pathogenesis.

Methodology Eligibility criteria included patients undergoing total abdominal/laparoscopic hysterectomy for endometrial cancer or benign indications. Microbiome swabs were collected along the female genital tract (FGT) (vagina, external cervical os, endometrium, fallopian tubes and ovaries) and rectum. The V1-V2 hypervariable regions of 16S rRNA genes were sequenced (Illumina MiSeq platform), data were analysed with Mothur software package and OTU taxonomies were determined. Benign and malignant endometrial organoids were cultured and treated with increasing concentrations (10\%, 20\%, $30 \%)$ of L. crispatus- conditioned media. Proliferation was assessed by the CellTiter-GLo ${ }^{\circledast 2}$ cell viability assay and cytokine/chemokine secretion (IL-1 $\beta$, IL-1ra, IL-2, IL-6, IL-8, IL-10, G- CSF, GM- CSF, IFN- $\gamma$, TNF- $\alpha$, CCL4/MIP1beta,
CCL5/RANTES) by the Magnetic Multiplex Cytokine Array (R\&D systems).

Result(s)* Sixty-one women were recruited; 37 had endometrial cancer and 24 were benign controls. We confirmed the presence of a genuine, low- abundance microbiome above background contamination in the endometrium, fallopian tubes and ovaries in a subset of benign and endometrial cancer patients, which was one- four orders of magnitude lower than the heavily colonised vagina, cervix and rectum. In 75\% (12/ 16) of benign patients, we found that the most abundant species of the lower genital tract could also be recovered from the whole length of the upper genital tract, while the microbial continuum was less cohesive in endometrial malignancy. We also demonstrated that Lactobacillus depletion and high microbial diversity along the genital tract are characteristic in endometrial cancer patients with concurrent enrichment of Porphyromonas, Prevotella, Peptoniphilus and Anaerococcus. Different histotypes and grades of endometrial cancer were not marked by microbial differences. L.crispatus, a FGT commensal that is depleted in endometrial cancer, was shown to reduce viability of endometrial cancer organoids at high concentrations and impact on cytokine secretion by benign and malignant endometrial organoids.

Conclusion* Endometrial cancer displays a distinct microbial signature. L.crispatus may exert an anti- proliferative effect in endometrial cancer and interfere with inflammatory pathways.

\section{INTERIM ANALYSIS OF 10-YEAR DATA REGARDING TREATMENT AND PROGNOSIS OF UTERINE CARCINOSARCOMA CASES ACROSS THE THAMES VALLEY CANCER ALLIANCE NETWORK}

${ }^{1}$ SL Smyth*, ${ }^{2} \mathrm{~A}$ Gkorila, ${ }^{3} \mathrm{~A}$ Mcculloch, ${ }^{4} \mathrm{P}$ Tupper, ${ }^{2} \mathrm{~A}$ Sattar, ${ }^{2} \mathrm{~N}$ Sadeghi, ${ }^{2} \mathrm{~S}$ Spencer, ${ }^{2} \mathrm{~K}$ Zarrindej, ${ }^{2} \mathrm{~V}$ Le Thanh, ${ }^{2} \mathrm{~J}$ Rencher, ${ }^{2} \mathrm{G}$ Sharma, ${ }^{2} \mathrm{~A}$ Kashif, ${ }^{2} \mathrm{M}$ Abdalla, ${ }^{1} \mathrm{~S}$ Addley, ${ }^{5} \mathrm{~S}$ Siddiki, ${ }^{2} \mathrm{M}$ Alazzam, ${ }^{2} \mathrm{H}$ Soleymani Majd. ${ }^{2}$ Churchill Hospital, Gynaecological Oncology, Oxford, UK; ${ }^{2} J o h n$ Raddliffe Hospital, Oxford, UK; ${ }^{3}$ Royal Berkshire Hospital, UK; ${ }^{4}$ Stoke Mandeville Hospital, UK; ${ }^{5}$ Great Western Hospital, UK

\subsection{6/ijgc-2021-ESGO.224}

Introduction/Background* Uterine Carcinosarcoma (UCS) comprises $<5 \%$ of uterine malignancies, accounting for $>15 \%$ associated mortality. With no established guidelines, we present our experience to determine optimal treatment and prognosis of UCS.

Methodology We conducted a multicentre retrospective cohort study, including all surgically managed UCS cases between March 2010 and January 2020. Data was collected on FIGO staging and post-operative management, recurrence and survival outcomes.

Result(s)* 82 (9.7\%) UCS cases were identified. Table 1 demonstrates case staging and management strategies. 23 patients underwent surgery alone due to poor performance status, comorbidities, age or treatment refusal. $15.8 \%$ had lymph nodes metastases, which was in keeping with the literature. Recurrence occurred in $47.8 \%$ and $77.8 \%$ of cases of early and late stage respectively; most frequently in the pelvis, but also commonly in the pelvic lymph nodes and chest. Both recurrence and subsequent death usually occurred within the first 1-2 years following treatment. This retrospective analysis explores for significance in overall and disease free survival between disease stage groups and treatment modalities using univariable and multivariable Cox regression models and Kaplan-Meier curves. 
Abstract 1019 Table 1 Treatment modalities of patients with UCS

Post-op FIGO stage

$46(56)$

Late-stage disease (FIGO III-IV) 36 (44)

Early-stage treatment

Surgery alone 16 (34.8)

Surgery and chemotherapy $\quad 4(8.7)$

Surgery and radiotherapy 17 (37)

Surgery and combined chemoradiotherapy

Late-stage treatment

Surgery alone

Surgery and chemotherapy

Surgery and radiotherapy

Surgery and combined chemoradiotherapy

7 (19.5)

$16(44.4)$

$4(11.1)$

$9(25)$

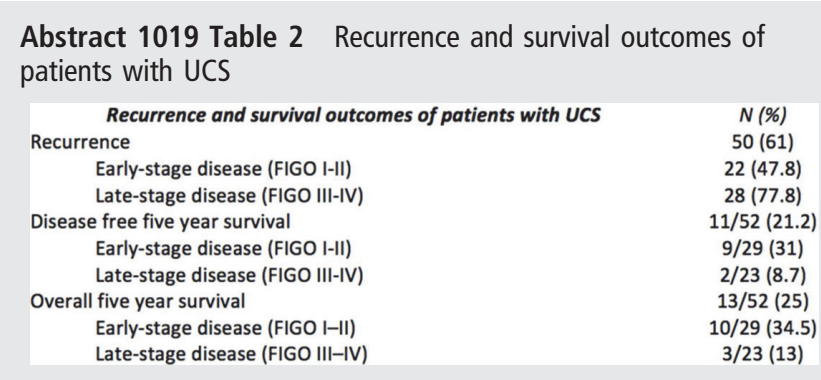

Conclusion* UCS is highly aggressive and has a poor prognosis. Significant rates of lymph node metastases have implications for adjuvant treatment in addition to high rates of relapse and distant metastases. Without an optimal treatment strategy established, discussions continue regarding adjuvant management as to potential improvements in prognosis. Whilst surgery remains the mainstay, multimodal treatment plans including combination of systemic chemotherapy followed by vaginal brachytherapy may be reasonable to address risks of both local relapse and distant metastases. In view of limited data and few prospective trials, we report on the results of our cancer network in contribution to evaluation of results of care in building a consensus for case management. We stress the importance of an individualised multidisciplinary team approach for management of UCS.

\section{ULTRASONOGRAPHIC ENDOMETRIAL THICKNESS AS A PREDICTOR OF THE RISK OF ENDOMETRIAL CANCER IN PATIENTS WITH POSTMENOPAUSAL BLEEDING}

M Tanturovski*, I Aluloski, S Stojchevski, V Jovanovska, M Stojovski. University Clinic of Gynecology and Obstetrics, Department of gynecologic oncology, Skopje, Macedonia

\subsection{6/ijgc-2021-ESG0.225}

Introduction/Background* Transvaginal ultrasound scanning (TVUS) to measure the endometrial thickness (ET) has historically been recommended as a first-line investigation of patients with postmenopausal bleeding. The aim of the study was to determine the diagnostic performance of endometrial thickness measured by transvaginal sonography in diagnosing endometrial cancer in patients presenting with post-menopausal bleeding.

Methodology The databases of the Department of Gynecological Oncology at the University Clinic of Gynecology and Obstetrics in Skopje, in the period January - December 2015 were searched in order to identify patients that underwent endometrial sampling due to newly-diagnosed postmenopausal bleeding. The following data were extracted from the patient records: age at sampling, age at menopause, parity, body mass index (BMI), American Society of Anesthesiologists physical status rating (ASA), history of hypertension and diabetes, endometrial thickness and the histology from the endometrial sampling. The endometrial thickness measurement was acquired in the mid sagittal plane at the thickest part. All patients underwent D\&C, with optional previous hysteroscopic evaluation (at he discretion of the attending) under general anesthesia.

Result(s)* A total of 158 patient records that met the criteria were identified. The prevalence of endometrial cancer was 15.2\%. Endometrial thickness was a statistically significant independent predictor of the presence of endometrial cancer and atypical endometrial hyperplasia (OR 1.19 95\% CI 1.091.29 for each $1 \mathrm{~mm}$ increase in thickness, $\mathrm{p}<0.001)$. The ROC curve analysis in our study had an AUC of 0.83 $(p<0.001)$ and identified a cut-off level for endometrial thickness of $8 \mathrm{~mm}$ which was associated to a sensitivity of $88.9 \%$, specificity of $65.6 \%$, PPV of $34.8 \%$ and NPV of $96.6 \%$ for the detection of endometrial cancer. Using a cutoff for endometrial thickness of $\leq 3 \mathrm{~mm}$ achieved $100 \%$ sensitivity.

Conclusion* None of the analyzed cut-off points for endometrial thickness achieved optimal diagnostic accuracy, as all cutoff values associated to sensitivity rates above $95 \%$ had false positive rates of over $60 \%$. Nevertheless, an endometrial thickness cut-off of $3 \mathrm{~mm}$, due to the associated high sensitivity, can safely be used to identify women with postmenopausal bleeding who are highly unlikely to harbor endometrial cancer and that can forego initial endometrial sampling.

\section{SENTINEL LYMPH NODE BIOPSY IN ENDOMETRIAL CANCER: DUAL INJECTION, DUAL TRACER. PELVIC AND PARA-AORTIC SLN DETECTION}

${ }^{1} \mathrm{~A}$ Torrent ${ }^{*},{ }^{2} \mathrm{~J}$ Amengual, ${ }^{3} \mathrm{C}$ Sampol, ${ }^{4} \mathrm{G}$ Matheu, ${ }^{5} \mathrm{M}$ Ruiz, ${ }^{6} \mathrm{~J}$ Rioja, ${ }^{7} \mathrm{O}$ Cordoba. ${ }^{1}$ Son Espases University Hospital, Gynecology Oncology, Palma, Spain; ${ }^{2}$ Son Espases University Hospital, Gynecologic oncology, Palma, Spain; ${ }^{3}$ Son Espases University Hospital, Nuclear Medicine, Palma, Spain; ${ }^{4}$ Son Espases University Hospital, Pathologucal Anatomy, Palma, Spain; ${ }^{5}$ Son Espases University Hospital, Gynecologic Oncology, Palma, Spain; ${ }^{6}$ Son Espases university Hospital, Gynecologic Oncology, Palma, Spain; ' ${ }^{7}$ Son Espases University Hospital, Gynecologic Oncology, Palma, Spain

\subsection{6/ijgc-2021-ESGO.226}

Introduction/Background* The aim of this study is to report the pelvic and Para-aortic SLN detection with a dual technique of indocyanine green (ICG) injection and Technetium 99 (Tc99) into the cervix and uterine fundus, in patients with Endometrial Cancer (EC)

Methodology Unicentric Prospective Study. 47 patients underwent laparoscopic surgery for EC in our center (with previous confirmative biopsy) between January 2019 and December 2020. A Dual Tracer was used (radiocolloid Technetium 99 (Tc99) and Indocyanine green (ICG) in 47 patients and a Dual Injection was performed (cervical and fundal) in 28 patients. Result(s)* The detection rates were: 95.7\%/45/47) for pelvic SLNs $(90.9 \%$ bilateral) in Tc99 injection and 100\% (44/44) for pelvic SLNs (88.6\%) for ICG tracer. A $6.38 \%$ (3) showed direct atypical drainage outside the standard field of pelvic lymphadenectomy (two presacral SLNs and one with direct drainage to 